\title{
COST-EFFECTIVENESS ANALYSIS OF CEFTRIAXONE AND NON-CEFTRIAXONE ON TYPHOID FEVER PATIENTS
}

\author{
CITRA SARI PURBANDINI, RANI SAURIASARI*
}

Department of Pharmacy, Faculty of Pharmacy, Universitas Indonesia, Depok, 16424, Indonesia. Email: rani@farmasi.ui.ac.id

Received: 20 June 2018, Revised and Accepted: 25 August 2018

ABSTRACT

Objective: This study aimed to evaluate the cost-effectiveness of ceftriaxone and non-ceftriaxone therapies in patients with typhoid fever.

Methods: The applied method was a cost-effectiveness analysis. Data were retrospectively collected, and sampling was performed using total sampling based on medical records and hospital information systems. Subjects were limited to patients diagnosed with typhoid fever and using ceftriaxone or non-ceftriaxone antibiotics. A total of 15 patients were investigated, comprising 10 patients on ceftriaxone and five patients using nonceftriaxone antibiotics. Effectiveness was evaluated by the length of hospitalization. The cost was a median of total costs, consisting of the cost of the drug, concomitant drugs, medical equipment, laboratory tests, doctor, health-care services, and hospitalization.

Results: The results showed the effectiveness of ceftriaxone ( $3.80 \pm 0.789$ days) did not differ with the non-ceftriaxone drugs ( $3.40 \pm 1.635$ days). However, the total cost of ceftriaxone (Rp 1,929,355) was less than that of non-ceftriaxone antibiotics (Rp 2,787,003). The average cost-effectiveness ratio of ceftriaxone group (Rp 507,725/effectiveness) was lower compared to the non-ceftriaxone (Rp 819,707/effectiveness).

Conclusions: This study results showed that ceftriaxone was more cost-effective than non-ceftriaxone antibiotics.

Keywords: Cost-effectiveness analysis, Typhoid fever, Hospitalization days, Ceftriaxone, Non-ceftriaxone.

(C) 2018 The Authors. Published by Innovare Academic Sciences Pvt Ltd. This is an open access article under the CC BY license (http://creativecommons. org/licenses/by/4. 0/) DOI: http://dx.doi.org/10.22159/ijap.2018.v10s1.18

\section{INTRODUCTION}

Typhoid fever is a bacterial infection caused by Salmonella enterica subspecies enterica serotype typhi or paratyphi A, B, or C [1]. Based on the WHO data, there are at least 21 million cases of typhoid fever and 222,000 deaths every year worldwide caused by the disease [2]. According to the data of Riskesdas (Indonesian Basic Health Research) in 2007, the prevalence of typhoid fever in Indonesia is $1.6 \%$ [3]. The rate of typhoid fever is estimated at 180.3/100,000 people per year [4].

In accordance with the Systematic Guideline for Typhoid Fever Control published in 2013, the first-line antimicrobial agents are chloramphenicol, ampicillin, amoxicillin (amoxicillin is safe to be used in pregnant patients), and trimethoprim-sulfamethoxazole. Other antibiotic options are also used in the treatment of typhoid fever to this day [5].

Ceftriaxone is an antibiotic often used for the treatment of typhoid fever [5]. Ceftriaxone may be used in cases of multidrug resistance (resistance to chloramphenicol, ampicillin, and cotrimoxazole) [6]. Ceftriaxone is well tolerated and can be used safely in children and adults [7]. Third-generation cephalosporins such as ceftriaxone or cefoperazone are generally well tolerated, although patients may show hypersensitivity reactions and can actively attack Salmonella bacteria [8].

In addition to third-generation cephalosporins, other classes of antibiotics such as quinolones and ciprofloxacin are also very effective against typhoid fever and other septic infections caused by Salmonella that passes through the bloodstream [9]. However, ciprofloxacin may have side effects on the liver and gastrointestinal tract that prolong hospitalization and may contribute to the development of antibiotic resistance, increasing the failure rate of fluoroquinolone therapy [10].

Based on the E-catalog website, ceftriaxone is relatively cheaper than non-ceftriaxone antibiotics [11]. This suggests that, in addition to the favorable efficacy of ceftriaxone over non-ceftriaxone antibiotics in the treatment of typhoid fever, the cost of ceftriaxone and non-ceftriaxone antibiotics varies enough.

Four analytical methods are used generally in pharmacoeconomic studies: Cost-minimalization analysis, cost-effectiveness analysis (CEA), cost-utility analysis, and cost-benefit analysis. These four analytical methods do not only consider the effectiveness, safety, and quality of the drugs compared but also the relevant economic aspects as well [12]. There are effectiveness and cost differences between ceftriaxone and non-ceftriaxone antibiotics; therefore, it is necessary to know which antibiotics will be more beneficial for hospitals and governments. CEA was selected for this study because it seemed to be the most appropriate and because it evaluates several drug treatments for the same condition [13]. CEA of antibiotics for typhoid fever patients has not been carried out before at the Cengkareng Regional General Hospital. Cengkareng Regional General Hospital was chosen as the site of this study because the hospital will prepare for the guidelines of antibiotic therapies. Therefore, this research can be used as a reference by policymakers in the hospital to determine antibiotics selection.

The effectiveness and total cost of treatment with ceftriaxone and non-ceftriaxone are not yet known, and which of the two treatments is more cost-effective in patients with typhoid fever need to be investigated as well. In general, this research aimed to assess the costeffectiveness of ceftriaxone and non-ceftriaxone antibiotics in the treatment of typhoid fever. In details, the purpose are (a) to assess the characteristics of patients participating in the study, (b) to analyze the effectiveness of ceftriaxone and non-ceftriaxone antibiotics in typhoid fever patients at Cengkareng Regional General Hospital in 2016, (c) to analyze the difference in total medical costs between groups of typhoid fever patients using ceftriaxone and non-ceftriaxone antibiotics at Cengkareng Regional General Hospital in 2016, and (d) to determine which antibiotic therapy is more cost-effective in the treatment of typhoid fever. 


\section{MATERIALS AND METHODS}

This study was an observational research with cross-sectional design and retrospective data retrieval on secondary data of patients. Secondary data were obtained from the patients' medical records and the information systems of Cengkareng Regional General Hospital in 2016. The results were presented descriptively and analytically. The research was conducted at Cengkareng Regional General Hospital, West Jakarta, the Special Capital Region of Jakarta from March to June 2017. The population of this study consisted of all patients suffering from typhoid fever and being treated with antibiotics at Cengkareng Regional General Hospital in 2016. The research sample consisted of all hospitalized patients with typhoid fever using ceftriaxone or nonceftriaxone antibiotics in 2016 and met the inclusion criteria of the research. Sampling was done by total sampling. The inclusion criteria of the study were as follows: Being diagnosed with typhoid fever and using ceftriaxone or non-ceftriaxone antibiotics (non-ceftriaxone antibiotics including cefoperazone and ciprofloxacin), at least 18 years of age, being otherwise cured of typhoid fever by a physician, using a payment system with BPJS (Social Insurance Administration Organization) and being guaranteed to receive care class Grade 3. Exclusion criteria were typhoid fever with comorbid illnesses such as typhoid encephalopathy, septic shock, peritonitis, typhoid hepatitis, typhoid pancreatitis, pneumonia, and dengue fever and incomplete, lost, and not legible medical records.

Data analysis was performed using the IBM Statistical Package for the Social Science software 20.0 with 95\% confidence level and $\alpha=0.05$. The data analyses included the CEA, univariate analysis to explain or describe the characteristics of each research variable, sample normality and homogeneity analysis, and bivariate analysis. CEA was conducted based on the calculation of the cost-effectiveness ratio (CER), the incremental cost-effectiveness ratio, and sensitivity analysis aimed to quantify the uncertainty of a variety of data used or generated in the pharmacoeconomic study).

\section{RESULTS AND DISCUSSION}

\section{Research subjects}

The study was conducted retrospectively for approximately 4 months (March 2017 to June 2017) based on the medical and administrative records of the Pharmacy Installation of Cengkareng Regional General Hospital. There were 119 patients suffering from typhoid fever throughout 2016. However, only 15 patients met the inclusion criteria. 104 patients were excluded: They had acute infectious diseases such as dengue fever, or their final medical diagnosis was not typhoid fever, or they were not treated in Grade 3. Ten patients were taking ceftriaxone antibiotics, and five patients were taking non-ceftriaxone antibiotics. Patients using non-ceftriaxone antibiotics included one patient using ciprofloxacin and four patients using cefoperazone. The patient group met the inclusion criteria of being diagnosed with typhoid fever and using ceftriaxone or non-ceftriaxone antibiotics. Therefore, only Ten patients in the ceftriaxone group and five patients in the non-ceftriaxone group were further analyzed.

\section{Patient characteristics}

More female than male patients participated in this research: eight women $(53.3 \%)$ and seven men $(46.7 \%)$. Five female $(50.0 \%)$ and five male $(50.0 \%)$ patients received ceftriaxone antibiotics, while two male $(40.0 \%)$ and three female $(60.0 \%)$ patients received nonceftriaxone antibiotics (60.0\%). Based on statistical analysis, there was no significant difference in sex between the two groups (Table 1).

The age of patients included in the study was mostly in the adult age category. The age range of patients in the ceftriaxone group was in the range of 20-55 years, whereas the patients of the non-ceftriaxone group were in the age range of 25-56 years. Based on the statistical analysis, the statistical significance was 0.371 . Therefore, it can be stated that there was no age difference between the two groups.
After the evaluation of patient residence data, it was found that 14 patients were from DKI Jakarta province and only one patient came from outside Jakarta Province (Banten Province).

Based on patient financing sources, Cengkareng Regional General Hospital serves patients with insurance (BPJS) and personal source (cash). However, because one of the inclusion criteria was using a payment system with BPJS, the pharmacoeconomic analysis used the perspective of the payer, that is, the hospital or the government as a health service provider. Hospital costs in both the ceftriaxone and the non-ceftriaxone groups were covered by the BPJS system. BPJS membership was divided into two groups: Contribution beneficiaries (PBI) and non-contribution beneficiaries (non-PBI) (BPJS of Health, 2013). According to Presidential Regulation No.12 of 2013 on Health Insurance, contribution beneficiaries of health insurance, hereinafter referred to as PBI health insurance, are the poor and disadvantaged participants of the Health Insurance Program. Nine patients were using the non-PBI system, while six patients were members of the PBI system.

The length of hospitalization was average for all patients. The average length of stay was 3.80 days in the ceftriaxone group and 3.40 days in the non-ceftriaxone group. Based on the statistical test, there was no difference in the average length of stay between the two patient groups.

\section{CEA}

Cost

A cost analysis was performed to determine the magnitude of the cost components involved in the treatment of ceftriaxone and nonceftriaxone groups by investigating the median of direct medical costs incurred by patients due to the use of ceftriaxone or non-ceftriaxone antibiotics, consisting of the cost of antibiotics, concomitant drugs, treatment, hospitalization, laboratory tests, and medical equipment. Information about the costs was obtained from SIM Cengkareng Regional General Hospital.

The cost of the treatment drug was the cost of intravenous or drip antibiotics. This cost was Rp 73,573.5 in the ceftriaxone group and Rp 397,024 in the non-ceftriaxone group. The difference is because that the non-ceftriaxone group was treated with more than one drug (cefoperazone and ciprofloxacin). Ceftriaxone is a first-line alternative as antimicrobials due to its extreme long half-life $(8 \mathrm{~h})$, and the use of them requires no costs expansion and no clinical experiments $[14,15]$. Therefore, the cost of non-ceftriaxone antibiotics was greater than the cost of ceftriaxone. In addition, the price of each drug was different, because the drugs used came from different factories. Based on the results of the non-parametric Mann-Whitney U-test, the statistical significance obtained was 0.001 . Therefore, it can be stated that there was a difference in antibiotic costs between the ceftriaxone group and the non-ceftriaxone group $(\mathrm{p}<0.05)$ (Fig. 1).

Other drug costs incurred by patients included the cost of antiemetic drugs for the reduction of nausea or vomiting, supplements or vitamins to boost immunity, parenteral infusion, and nutrition supplies. The cost of concomitant drugs was Rp 662,025.5 in the ceftriaxone group and $\mathrm{Rp} 804,009$ in the non-ceftriaxone group. Based on the non-parametric Mann-Whitney U-test, the significant difference was 0.679 ( $p>0.05$ ). Therefore, it can be stated that there was no difference in the cost of concomitant drugs between the two groups.

The cost of medical equipment related to the use of intravenous antibiotics was calculated and compared between the ceftriaxone and non-ceftriaxone groups. The cost of medical equipment included the purchase of medical equipment to be used during hospitalization, such as $10 \mathrm{cc}$ syringes, 3 cc syringes, 5 cc syringes, 1 cc syringes, needles, gloves, and others. The cost of medical equipment was Rp 81,256 in the ceftriaxone group and Rp 95,970 in the non-ceftriaxone group. Based on the non-parametric Mann-Whitney U-test, the significant difference was $0.679(p>0.05)$. Therefore, it can be stated that there was no difference in the cost of medical equipment between the two groups. 


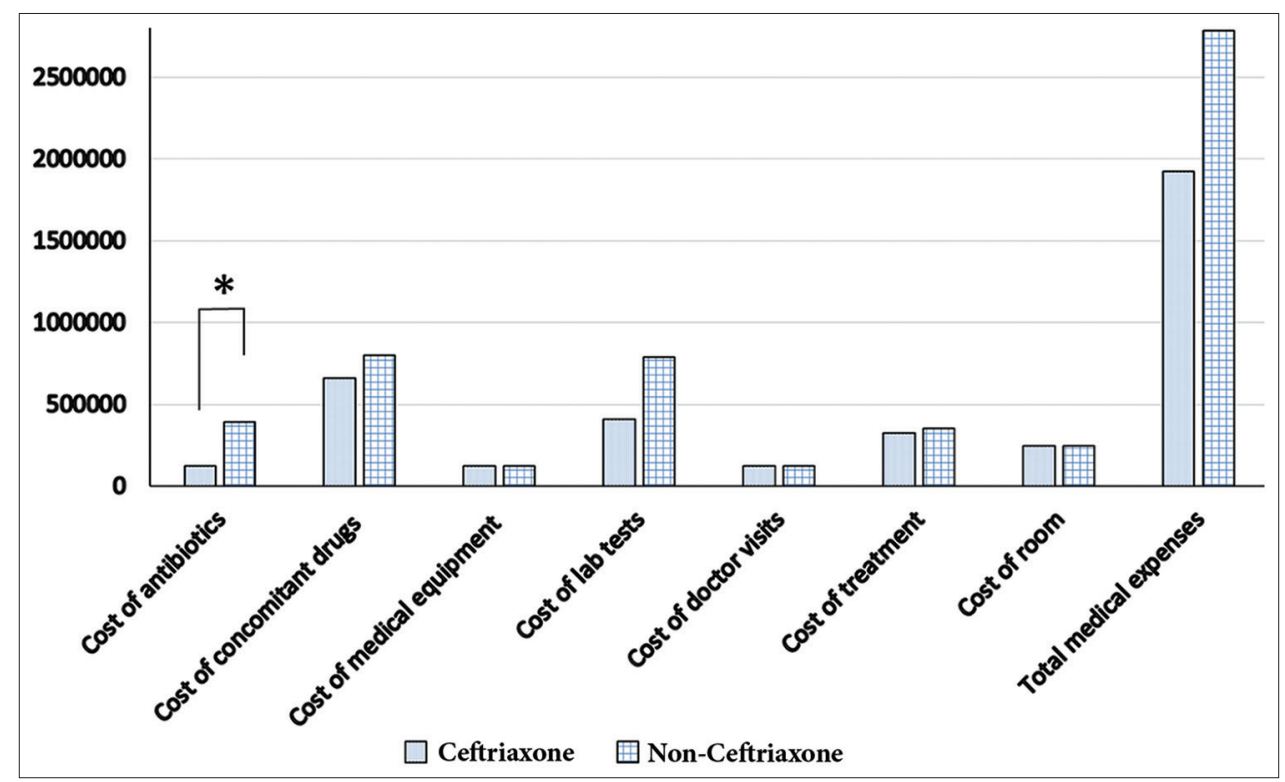

Fig. 1: Comparison of direct medical costs between typhoid fever patients using ceftriaxone and non-ceftriaxone antibiotics at Cengkareng Regional General Hospital in 2016

Table 1: Characteristics of patients using ceftriaxone and non-ceftriaxone antibiotics at Cengkareng Regional General Hospital in 2016

\begin{tabular}{llll}
\hline Characteristics/variables & Ceftriaxone $\mathbf{n = 1 0}(\mathbf{\%})$ & Non-ceftriaxone $\mathbf{n = 5}$ (\%) & $\mathbf{p}$ \\
\hline Gender & & & $1.000^{\mathrm{a}}$ \\
$\quad$ Male & $5(50.0)$ & $2(40.0)$ & $3(60.0)$ \\
$\quad$ Female & $5(50.0)$ & $42 \pm 14.95(25-56)$ & $0.371^{\mathrm{b}}$ \\
Age (years old) & $39 \pm 12.61(20-55)$ & $3(60.0)$ & $1.000^{\mathrm{a}}$ \\
BPJS & $6(60.0)$ & $2(40.0)$ \\
$\quad$ Non-PBI & $4(40.0)$ & $4(80.0)$ & $0.333^{\mathrm{a}}$ \\
PBI & $10(100.0)$ & $1(20.0)$ & \\
Origin & $0(0.0)$ & \\
DKI Jakarta & & \\
$\quad$ Outside Jakarta &
\end{tabular}

Laboratory test costs were costs incurred by patients due to laboratory examinations for diagnostic purposes, monitoring of side effects and therapeutic progress, or determining the outcome of therapy. Laboratory tests included hematological examination such as Hema I $(\mathrm{Hb}$, hematocrit, leukocyte, and platelets), complete blood count (LED, Hb, $\mathrm{Ht}$, erythrocytes, leukocytes, platelets, mean corpuscular volume, mean corpuscular hemoglobin [MCH], MCHC concentration, and count type), immunoserology examination using the Widal test, blood chemistry examination (aminotransferase, alanine aminotransferase, gammaglutamyl transferase, albumin, urea, creatinine, and uric acid), electrolyte examination, blood glucose examination, and radiological examination of the chest (PA/AP/RLD views). Microbiological examinations involving blood, urine, and feces culture tests, as well as a resistance test, were also performed. The cost of laboratory tests was Rp 412,500 in the ceftriaxone group and $\mathrm{Rp} \mathrm{795,000} \mathrm{in} \mathrm{the} \mathrm{non-ceftriaxone} \mathrm{group.} \mathrm{Based} \mathrm{on} \mathrm{the} \mathrm{non-}$ parametric Mann-Whitney U-test, the significant difference was 0.513 ( $>0.05)$. Therefore, it can be stated that there was no difference in the cost of laboratory tests between the two groups.

The cost of doctor visits was the cost of doctor visits during the time of hospitalization. The cost of doctor visits was Rp 120,000 in the ceftriaxone group and Rp 90,000 in the non-ceftriaxone group. Based on the non-parametric Mann-Whitney U-test, the significant difference was $0.513(p>0.05)$. Therefore, it can be stated that there was no difference in the cost of doctor visits between the two groups.

The cost of treatment was the cost incurred by patients due to medical treatment during the hospitalization. The treatment included partial care, infusion installation, catheter installation, electrocardiogram monitor installation, and injection in the ER. The cost of treatment was Rp 330,000 in the ceftriaxone group and $\mathrm{Rp} 355,000$ in the non-ceftriaxone group. Based on the non-parametric Mann-Whitney U-test, the significant difference was 0.513 ( $p>0.05)$. Therefore, it can be stated that there was no difference in the cost of treatment between the two groups.

The cost of the room was the cost incurred by patients for hospital accommodation during the hospitalization. The cost of the room was $\mathrm{Rp}$ 250,000 in both the ceftriaxone group and the non-ceftriaxone group. Based on the non-parametric Mann-Whitney U-test, the significant difference was 0.679 ( $p>0.05)$. Therefore, it can be stated that there was no difference in the cost of room between the two groups.

The total cost of treatment was calculated from the sum of all direct medical cost components, including the cost of antibiotics, concomitant drugs, medical equipment, laboratory tests, treatment, hospitalization, and doctors' services for each patient. Next, the total cost of treatment was obtained for each group. The total cost of treatment was Rp $1,929,355$ in the ceftriaxone group and Rp 2,787,003 in the nonceftriaxone group. It suggested that the total cost of treatment in the non-ceftriaxone group was higher than in the ceftriaxone group. Based on the non-parametric Mann-Whitney U-test, the significant difference was $0.099(p>0.05)$. Therefore, it can be stated that there was no difference in the total cost of treatment between the ceftriaxone and non-ceftriaxone groups.

\section{Effectiveness}

The effectiveness of the treatment was measured as the length of hospitalization. Data on effectiveness were obtained from the patients' 
Table 2: Comparison of treatment effectiveness of patients with typhoid fever using ceftriaxone and non-ceftriaxone antibiotics at Cengkareng Regional General Hospital in 2016

\begin{tabular}{llll}
\hline Group of antibiotics & The number of patients with hospitalization $\leq \mathbf{5}$ days (\%) & Length of hospitalization & $\mathbf{p}$ \\
\hline Ceftriaxone $(\mathrm{n}=10)$ & 100 & $3.80 \pm 0.789(3-5$ days $)$ & $3.40 \pm 1.635(3-4$ days $)$ \\
Non-ceftriaxone $(\mathrm{n}=5)$ & 100 &
\end{tabular}

Table 3: Comparison of the CER in typhoid fever patients using ceftriaxone and non-ceftriaxone antibiotics at Cengkareng Regional General Hospital in 2016

\begin{tabular}{llll}
\hline $\begin{array}{l}\text { Group of } \\
\text { antibiotics }\end{array}$ & $\begin{array}{l}\text { Effectiveness } \\
\text { (day) }\end{array}$ & $\begin{array}{l}\text { Total } \\
\text { cost (Rp) }\end{array}$ & CER \\
\hline $\begin{array}{l}\text { Ceftriaxone } \\
\text { Non-ceftriaxone }\end{array}$ & 3.80 & $1,929,355$ & $507,725.00$ \\
antibiotics & 3.40 & $2,787,003$ & $819,706.76$ \\
\hline
\end{tabular}

CER: Cost-effectiveness ratio

Table 4: Result of the sensitivity analysis

\begin{tabular}{|c|c|c|}
\hline Description & Ceftriaxone $(n=10)$ & Non-ceftriaxone antibiotics $(n=5)$ \\
\hline Total direct cost per patient increased & Total cost per patient & Total cost per patient \\
\hline $0 \%$ of total cost $/$ patient & Rp $1,929,355$ & $\operatorname{Rp} 2,787,003.00$ \\
\hline $5 \%$ of total cost/patient & Rp 2,025,822.75 & Rp 2,926,353.15 \\
\hline $10 \%$ of total cost/patient & Rp 2,122,290.50 & Rp 3,065,703.30 \\
\hline $15 \%$ of total cost/patient & $\operatorname{Rp} 2,218,758.25$ & $\operatorname{Rp} 3,205,053.45$ \\
\hline \multicolumn{3}{|l|}{ Effectiveness (E) } \\
\hline Length of hospitalization & $3.80 \pm 0.789$ & $3.40 \pm 1.635$ \\
\hline \multicolumn{3}{|l|}{ CER improved } \\
\hline $0 \%$ of initial CER & Rp 507,735 & Rp 819,706.76 \\
\hline $5 \%$ of initial CER & $\operatorname{Rp} 533,111.25$ & $\operatorname{Rp} 860,692.10$ \\
\hline $10 \%$ of initial CER & Rp 558,497.50 & Rp 901,677.44 \\
\hline $15 \%$ of initial CER & $\operatorname{Rp} 583,883.75$ & $\operatorname{Rp} 942,662.78$ \\
\hline Position & Dominant & Dominated \\
\hline
\end{tabular}

medical records and the information from systems of Cengkareng Regional General Hospital (Table 2).

The percentage effectiveness of ceftriaxone did not differ with the nonceftriaxone in this study; even previous studies showed that ceftriaxone was more effective for typhoid fever treatment and safe to use for children and adults [7,16]. Ceftriaxone was also preferred because it was highly efficient as a typhoid fever treatment and had few side effects $[17,18]$.

\section{CER}

The calculation of the CER was carried out by comparing the total cost of effective treatment in each treatment group [19]. The total cost included in the calculation was the total cost of treatment in each treatment group. The effectiveness included was the average length of hospitalization.

CER of each antibiotic group showed that the CER of the non-ceftriaxone group was greater (Rp 819,707/effectiveness) compared to the ceftriaxone group (Rp 507,725/effectiveness) (Table 3). The costeffective selection of drugs allowed the health-care funds to be used more rationally; therefore, the quality and scope of services could be improved [12]. Based on CER calculations, it was found that the effectiveness of ceftriaxone was higher with a lower total cost compared to nonceftriaxone antibiotics. Both groups of antibiotics met the requirements of a cost-effective program. However, the program chosen and the most cost-effective program was not always the lower cost alternative [16].

\section{Sensitivity analysis}

Sensitivity analysis was the process of testing the robustness of the economic evaluation by making changes to the results of the research $[12,16]$. The sensitivity analysis conducted in this research was a one-way sensitivity analysis, that is, a simulation of cost value changes with fixed effectivity value. The sensitivity analysis was carried out by simulating an increase in total medical expenses by increasing the values by $5 \%, 10 \%$, and $15 \%$ (Table 4 ).

Based on the calculation results of the above sensitivity analysis, the total value of direct cost and the constant CER of ceftriaxone were lower than the same values calculated for the non-ceftriaxone antibiotics, although the total value of medical cost directly increased. Therefore, it can be concluded that the treatment carried out with ceftriaxone was more dominant than the treatment with non-ceftriaxone antibiotics.

\section{CONCLUSIONS}

Based on the results of this study, it can be concluded that the average CER of the ceftriaxone group (Rp 507,725/effectiveness) was lower compared to the non-ceftriaxone (Rp 819,707/effectiveness). This study results showed that ceftriaxone was more cost-effective than non-ceftriaxone antibiotics.

\section{CONFLICTS OF INTEREST}

None declared.

\section{REFERENCES}

1. NICD: Typhoid : NICD Recommendations for Diagnosis, Management and Public Health Response. South Africa: The National Institute for Communicable Diseases; 2016.

2. WHO. Typhoid in Vaccines and Disease. Washington, DC: WHO; 2015.

3. Agency for Health Research and Development. Indonesian Basic 
Health Research 2007. Jakarta: Department of Health, Republic of Indonesia; 2008

4. Berger S. Typhoid and Enteric Fever: Global Status. California: GIDEON Informatics, Inc.; 2015.

5. Fithria RF, Damayanti K, Fauziah P. Differences in the Effectiveness of Antibiotics in the Treatment of Typhoid Fever in Bancak, Semarang, 2014. Proceeding of National Seminar: Opportunity of Herbal as Alternative Medicine; 2015. p. 1-6.

6. Naveed A, Ahmed Z. Treatment of typhoid fever in children: Comparison of efficacy of ciprofloxacin with ceftriaxone. J Middle East North Afr Sci 2015;12 Suppl 6:346-55.

7. Rani MU. Comparative study of efficacy of cefuroxime and ceftriaxone in enteric. Fever 2015;14 Suppl 1:27-32.

8. Stoesser N, Eyre D, Basnyat B, Parry C. Treatment of enteric fever (typhoid and paratyphoid fever) with third and fourth generation cephalosporins (Protocol). Cochrane Database Syst Rev 2013;2013:CD010452.

9. Sköld O. Antibiotics and Antibiotics Resistance. Canada: John Wiley and Sons, Inc.; 2011.

10. Nelwan RH. Levofloxacin : Today's Choice for the Treatment of Typhoid Fever? An Illustrative Case Report from Indonesia; n.d. p. 41-4.

11. Government of KLPB. Drugs Product Catalog; 2017. Available from: http://www.e-katalog.lkpp.go.id.
12. Ministry of Health, Republic of Indonesia. Guidelines for the Application of the Economic Pharmacy. Jakarta: Ministry of Health; 2013.

13. Baldi A. Pharmacoeconomics: Principles, methods and economic evaluation of drug therapies. Ph Tech Med 2017;2 Suppl 5:362-9.

14. Nimin P, Santhosh KV. A comparative study on drug use of antimicrobial agents in medical surgical intensive care units of a tertiary care hospital. Asian J Pharm Clin Res 2018;11:310-3.

15. Mohd YK, Maryada R, Raj KR, Umesh KB. A review-ceftriaxone for life. Asian J Pharm Res 2017;7:35-48.

16. Limpitikul W, Singchungchai P. Costs and effectiveness of ciprofloxacin and ceftriaxone in treatment of typhoid fever in children in Thailand. Int J Public Health 2012;3 Suppl 1:192-7.

17. Choudhary T. Comparison of injectable ciprofloxacin and injectable ceftriaxone for the treatment of hospitalized patients with enteric fever. Ann Pak Int Med Sci 2014;10 Suppl 4:182-6.

18. Teti ST, Dewi RM. The rationality of antibiotics therapy toward childern with typhoid fever at M.M. Dunda Hospital. Asian J Pharm Clin Res 2018;11:185-8.

19. Trask LS. Pharmacoeconomics: Principles, methods, and applications. In: Di Piro JT, Talbert RL, Yee GC, Matzke GR, Wells BG, Posey LM, editors. Pharmacotherapy: A Pathophysiologic Approach. 8th ed. New York: McGraw-Hill Companies, Inc.; 2011. 